\title{
A new class of metric $f$-manifolds
}

\section{Pablo Alegre ${ }^{1}$, Luis M. FernándeZ ${ }^{2}$ and Alicia Prieto-Martín ${ }^{2}$}

\section{ABSTRACT.}

We introduce a new general class of metric $f$-manifolds which we call (almost) trans- $S$-manifolds and includes $S$-manifolds, $C$-manifolds, $s$-th Sasakian manifolds and generalized Kenmotsu manifolds studied previously. We prove their main properties and we present many examples which justify their study.

\section{REFERENCES}

[1] Bhatt, L. and Dube, K. K., Semi-invariant submanifolds of r-Kenmotsu manifolds, Acta Cienc. Indica Math., 29 (2003), No. 1, 167-172

[2] Blair, D. E., Geometry of manifolds with structural group $\mathcal{U}(n) \times \mathcal{O}(s)$, J. Differential Geom., 4 (1970), 155-167

[3] Blair, D. E., Riemannian Geometry of Contact and Symplectic Manifolds, 2nd. Ed., Progress in Mathematics, Vol. 209, Birkhäuser, New York, 2010

[4] Blair, D. E and Oubiña, J. A., Conformal and related changes of metric on the product of two almost contact metric manifolds, Publ. Mat., 34 (1990), 199-207

[5] Falcitelli, M. and Pastore, A. M., $f$-structures of Kenmotsu type, Mediterr. J. Math., 3 (2006), 549-564

[6] Fernández, L. M., Variedades con K-estructuras. Subvariedades, Ph. D. Thesis, University of Sevilla, Sevilla, 1987

[7] Goldberg, S. I. and Yano, K., On normal globally framed f-manifolds, Tohoku Math. J., 22 (1970), No. 2, 362-370

[8] Hasegawa, I., Okuyama, Y. and Abe, T., On p-th Sasakian manifolds, J. Hokkaido Univ. Educ. Nat. Sci. Section II A, 37 (1986), No. 1, 1-16

[9] Olszak, Z., Curvature properties of quasi-Sasakian manifolds, Tensor (N.S.), 38 (1982), 19-28

[10] O'Neill, B., Semi-Riemannian Geometry with Applications to Relativity, Pure and Applied Mathematics, Vol. 103, Academic Press, New York, 1983

[11] Oubiña, J. A., New classes of almost contact metric structures, Publ. Mat., 32 (1985), 187-193

[12] Suguri, S. and Nakayama, S., D-conformal deformations on almost contact metric structure, Tensor (N.S.), 28 (1974), 125-129

[13] Tanno, S., The topology of contact Riemannian manifolds, Illinois J. Math., 12 (1968), 700-717

[14] Turgut Vanli, A. and Sari, R., Generalized Kenmotsu manifolds, Comm. Math. Appl., 7 (2016), No. 4, 311-328

[15] Yano, K., On a structure defined by a tensor field $f$ of type $(1,1)$ satisfying $f^{3}+f=0$, Tensor, 14, (1963), 99-109

DEPARTAMENTO DE ECONOMÍA

MÉTOdos CuAntitativos e Historia de la ECONOMía

Universidad PABlo de OlaVide, CTRA. DE UtRERA, KM. 1

41013 SEVILLA, SPAIN

E-mail address: psalerue@upo.es

Received: 15.01.2018; In revised form: 30.05.2018; Accepted: 06.06.2018

2010 Mathematics Subject Classification. 53C15, 53C25, 53C99.

Key words and phrases. Almost trans-S-manifold, trans-S-manifold, generalized D-conformal deformation, warped product.

Corresponding author: Luis M. Fernández; lmfer@us.es 
Departamento DE GeOMETRÍa Y TOPOLOGía

FACULTAD DE MATEMÁticas

UNIVERSIDAD DE SEVILLA

C./ TARFia, S.N. 41012 Sevilla, Spain.

E-mail address: Imfer@us.es

E-mail address: aliciaprieto@us.es 